\title{
Reconstruction of fiber grating refractive-index profiles from complex Bragg reflection spectra
}

\author{
Ding-Wei Huang and Chih-Chung Yang
}

\begin{abstract}
Reconstruction of the refractive-index profiles of fiber gratings from their complex Bragg reflection spectra is experimentally demonstrated. The amplitude and phase of the complex reflection spectrum were measured with a balanced Michelson interferometer. By integrating the coupled-mode equations, we built the relationship between the complex coupling coefficient and the complex reflection spectrum as an iterative algorithm for reconstructing the index profile. This method is expected to be useful for reconstructing the index profiles of fiber gratings with any apodization, chirp, or dc structures. An apodized chirped grating and a uniform grating with a depression of index modulation were used to demonstrate the technique. (C) 1999 Optical Society of America

OCIS codes: $050.2770,060.2340$.
\end{abstract}

\section{Introduction}

Fiber gratings fabricated with UV laser exposure on photosensitive optical fiber have been widely used for optical filtering,,$^{1,2}$ sensing, ${ }^{3}$ dispersion compensation, ${ }^{4,5}$ etc. Bragg reflection characteristics of a fiber grating, including amplitude and phase, are controlled by its longitudinal refractive-index distribution, i.e., the local grating period and the depth of effective index modulation. Therefore, the retrieval of the longitudinal index distribution is crucially important for controlling the characteristics of a fiber grating. Several studies have been conducted for this purpose. The technique of side diffraction ${ }^{6}$ has been used to determine the index profile. However, this technique suffers from the disadvantage of strict alignment of the probe laser beam and the lack of information of background index change and grating chirp. Also, the spatial resolution is limited by the size of the probe beam. The technique of side scattering can be used to measure the grating chirp. ${ }^{7}$ However, the spatial resolution is limited by the detection system. An algorithm was proposed to retrieve the index profiles by processing the data from

\footnotetext{
The authors are with the Department of Electrical Engineering and Graduate Institute of Electro-Optical Engineering, National Taiwan University, 1 Roosevelt Road, Section 4, Taipei, Taiwan. The e-mail address for C. C. Yang is ccy@cc.ee.ntu.edu.tw.

Received 4 January 1999; revised manuscript received 29 March 1999.

0003-6935/99/214494-06\$15.00/0

(C) 1999 Optical Society of America
}

optical coherence-domain reflectometry (OCDR). ${ }^{8}$ However, no experimental work was reported based on the proposed method. Besides, it is difficult to obtain the required phase information from the OCDR measurement. Recently, it was reported that the phase information could be obtained from reflectivity data through the Hilbert (or Wiener-Lee) transformation without direct measurement.9-11 However, this process has some limitation, i.e., the grating must be symmetric or the reflection response must be a minimum phase-shift function. Therefore, without direct measurement of phase, it is difficult to retrieve the index profiles of gratings with apodization, chirp, or dc component structures.

Here we report our results of retrieving the index profiles of fiber gratings based on a novel technique. We measure both intensity and phase of Bragg reflection from the fiber grating with an interferometric setup. Then, with a simple algorithm, we can retrieve index profiles of fiber gratings with wellbehaved apodization, chirp, or dc structures under the following conditions: (1) the period of the fiber grating is either uniform or linearly chirped; (2) the envelope of index change is not exactly a linear function. Although our basic theory is the same as that in Ref. 8, we provide more concrete discussions on the required procedures for retrieving the apodization, chirp, and dc structures. Meanwhile, our experimental implementation demonstrates the feasibility of this algorithm. Two fiber grating samples were tested to demonstrate the capability of the technique.

In Section 2 we discuss the theoretical background of the technique. In Section 3 we describe the pro- 
cedure for reconstructive refractive-index distribution. We provide the reconstruction results of the two fiber gratings in Section 4. Finally, a discussion and the conclusion are presented in Section 5 .

\section{Theoretical Analysis}

For fiber gratings fabricated with an UV laser, the index profile can be described by

$$
n(z)=n_{o}+\delta n(z)\left\{b+\cos \left[\frac{2 \pi}{\Lambda_{o}} z+\phi(z)\right]\right\},
$$

where $n_{0}$ is the effective refractive index of the fiber before UV exposure, $\delta n(z)$ is the envelope of index change, $b$ is the background component of the index change $[b \delta n(z)$ is the dc component of the index change], $\Lambda_{o}$ is the nominal grating period, and $\phi(z)$ describes the grating chirp. For a linearly chirped grating, the phase term has the form

$$
\phi(z)=\frac{-\pi}{\Lambda_{o}} \alpha z^{2}
$$

where $\alpha=0$ for a uniform grating. If we define the complex amplitudes $A(z, v)$ and $B(z, v)$ for the forward propagating wave field $E_{+}(z, v)$ and the backward propagating wave field $E_{-}(z, v)$ in the grating as

$$
\begin{aligned}
& E_{+}(z, v)=A(z, v) \exp [-j \sigma(z) z], \\
& E_{-}(z, v)=B(z, v) \exp [j \sigma(z) z],
\end{aligned}
$$

respectively, where

$$
\sigma(z)=\frac{2 \pi}{\lambda} \delta n(z) b
$$

is a slowly varying propagation constant corresponding to the dc component of the index change, the coupled-mode equation can be reduced to ${ }^{12}$

$$
\begin{aligned}
& \frac{\mathrm{d} A}{\mathrm{~d} z}=\tilde{\kappa}^{*} B \exp (j 2 \pi v z), \\
& \frac{\mathrm{d} B}{\mathrm{~d} z}=\tilde{\kappa} A \exp (-j 2 \pi v z),
\end{aligned}
$$

Here,

$$
v=\frac{\beta}{\pi}-\frac{1}{\Lambda_{o}}=2 n_{o}\left(\frac{1}{\lambda}-\frac{1}{\lambda_{B_{o}}}\right)
$$

is the frequency detuning, $\beta=2 \pi n_{o} / \lambda$ is the background propagation constant, and $\lambda_{B_{o}}=2 n_{o} \Lambda_{o}$ is the central Bragg wavelength. Meanwhile, the complex coupling coefficient $\tilde{\kappa}(z)$, which contains the index change information, is given by

$$
\tilde{\kappa}(z)=j \frac{\pi}{\lambda} \delta n(z) \exp \{-j[2 \sigma(z) z+\phi(z)]\} .
$$

By direct integration of Eqs. (6) and (7), the backward propagating field at the input end, $B(0, v)$, can be

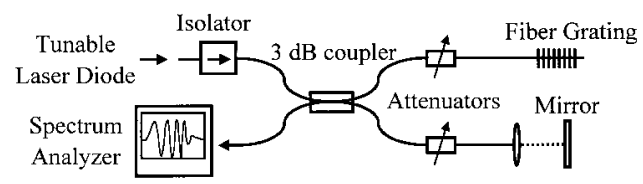

Fig. 1. Balanced Michelson interferometer for measurement.

expressed as an integral of the complex coupling coefficient multiplied by the field distribution in the grating. This expression can be further reduced to the relation between the complex reflection spectrum and the complex coupling coefficient as

$$
\begin{aligned}
r(0, v)= & -\int_{0}^{L}\left\{\tilde { \kappa } ( z ) \left[1+\int_{0}^{z} \tilde{\kappa} *(z) r\left(z^{\prime}, v\right)\right.\right. \\
& \left.\left.\times \exp \left(j 2 \pi v z^{\prime}\right) \mathrm{d} z^{\prime}\right]\right\} \exp (-j 2 \pi v z) \mathrm{d} z
\end{aligned}
$$

where $r(z, v)=B(z, v) / A(0, v)$ is the normalized backward propagating wave field and $r(0, v)$ is the complex reflection coefficient measured at the input end of the fiber grating. Equation (10) shows that the complex reflection spectrum is the Fourier transform of the quantity within braces on the right-hand side of Eq. (10). After taking the inverse Fourier transform of Eq. (10), we can obtain an iterative relation as

$$
\tilde{\kappa}_{m+1}(z)=-\mathscr{F}^{-1}\{r(0, v)\}-\mathscr{F}^{-1}\left\{p_{m}(v)\right\},
$$

where

$$
\begin{aligned}
p_{m}(v)= & \int_{0}^{L} \tilde{\kappa}_{m}(z) \int_{0}^{z} \tilde{\kappa}_{m} *\left(z^{\prime}\right) r_{m}\left(z^{\prime}, v\right) \\
& \times \exp \left[j 2 \pi v\left(z^{\prime}-z\right)\right] \mathrm{d} z^{\prime} \mathrm{d} z .
\end{aligned}
$$

Meanwhile, $\mathscr{F}$ denotes the Fourier transformation operator and the subscript $m=0,1,2, \ldots$ is the iteration number. We calculated the normalized backward propagating field $r_{m}(z, v)$ by using Eqs. (6) and (7) with $\tilde{\kappa}(z)=\tilde{\kappa}_{m}(z)$ at each iteration. Equation (11) describes the algorithm for reconstructing the complex coupling coefficient $\tilde{\kappa}(z)$ from the complex reflection spectrum. The initial conditions in the algorithm are set to be $\tilde{\kappa}_{0}(z)=0$ and $r_{0}(z, v)=0$. Hence, with the procedure described above, we can obtain $\tilde{\kappa}(z)$ from the measured complex reflection spectrum from which the index profile of the fiber grating can be consequently obtained through Eq. (9).

\section{Reconstruction Procedures}

Figure 1 shows the experimental setup that we used to measure the amplitude and phase of the Bragg reflection coefficient. The system is a balanced Michelson interferometer with a test arm connected to the fiber grating to be characterized and a freespace reference arm in which the signal is retroreflected by a gold-coated mirror. A tunable laser diode with a wavelength resolution of $0.001 \mathrm{~nm}$ was 
used as the light source. We measured the fiber Bragg reflection, interference, and reference intensities by appropriately blocking light with the two attenuators. The Bragg reflection, interference, and reference intensities, normalized by the input intensity, have the following relationships represented by $I_{\text {reflect }}, I_{\text {inter }}$, and $I_{\text {ref }}$, respectively:

$$
\begin{aligned}
|r| & =\sqrt{I_{\text {reflect }},} \\
I_{\text {inter }} & =I_{\text {ref }}+I_{\text {reflect }}+2 \sqrt{I_{\text {ref }} I_{\text {reflect }}} \cos \left(\phi_{r}\right),
\end{aligned}
$$

Meanwhile, $|r|$ and $\phi_{r}$ are the amplitude and phase of the complex reflection coefficient at the position $z=0$. By measuring the spectra of $I_{\text {reflect }}, I_{\text {inter }}$ and $I_{\text {ref }}$, the phase part of the complex reflection coefficient can be retrieved through Eqs. (13) and (14) with a suitable curve fitting as described in Ref. 13. Note that the retrieved phase can be either positive or negative from Eq. (14). Because of the time reversal property of the Fourier transform of a conjugate signal, opposite signs of phase could lead to the reconstructed index distribution either in the region $z \geq 0$ or $z<0$. To avoid such ambiguity, we can carefully arrange the interferometer so that the optical path in the reference arm is equal to or shorter than that in the test arm. In other words, the interferometer is set as a causal system. Hence, we can choose the correct sign for the retrieved phase of the reflection coefficient, which ensures that the reconstructed index distribution is located in the region $z \geq 0$.

After we have the complex reflection coefficient $r(z$, $v)$, Eq. (11) can be used to reconstruct the complex coupling coefficient $\tilde{\kappa}(z)$. After we have $\tilde{\kappa}(z)$, we can then obtain $\delta n(z)$ by taking the absolute values of both sides of Eq. (9), i.e.,

$$
\delta n(z)=|\tilde{\kappa}(z)|(\lambda / \pi) .
$$

Note that the dc component of index change and grating chirp are mixed in the phase of the complex coupling coefficient. We can separate these two quantities with the following assumptions: (1) the period of the fiber grating is either uniform or linearly chirped and (2) $\delta n(z)$ is not a linear function. With these assumptions, the first-order derivative of the phase of the complex coupling coefficient can be a linear combination of the dc component of index change and grating chirp with the two parameters $b$ and $\alpha$ as

$$
\frac{\mathrm{d} \phi_{\tilde{\kappa}}(z)}{\mathrm{d} z}=\frac{\mathrm{d} \arg [\tilde{\kappa}(z)]}{\mathrm{d} z}=-b \frac{2 \pi}{\lambda} \delta n(z)+\frac{2 \pi}{\Lambda_{0}} \alpha z .
$$

We can then use a linear programming method to fit Eq. (16) to separate these two quantities. Figure 2 shows the retrieving algorithm procedures.

\section{Reconstruction Results}

In our experiment we measured two fiber gratings with different index profiles. First, we measured a fiber grating fabricated with a chirped phase mask (chirp of the Bragg wavelength is $0.02 \mathrm{~nm} / \mathrm{mm}$ ).

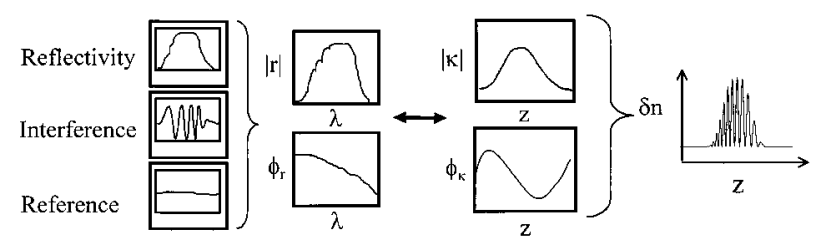

Fig. 2. Procedures of the retrieving algorithm.

Apodization was assumed to be due to the nearly Gaussian profile of the UV laser beam that we used. Figure 3 shows the measured values of $I_{\text {reflect }}, I_{\text {inter }}$, and $I_{\text {ref }}$ of this grating. Note that the structure on the short wavelength side of the reflectivity spectrum, $I_{\text {reflect }}$, is known as self-chirping, which implies that the index profile might have a nonconstant dc component. Figure 4 shows the retrieved amplitude and phase of the reflection coefficient (the continuous curve in the lower portion is drawn to fit the data points of filled circles). After several iterations of the reconstructing algorithm, we obtained satisfactory results for the reconstructed complex coupling coefficient. Figure 5 shows the amplitude and phase of the reconstructed coupling coefficient of this grating, as functions of position in the grating. The reconstructed amplitude of the coupling coefficient after each iteration is also shown. The curve at the top represents the result after the last iteration. The result implies a nearly $\exp \left(-|z|^{3}\right)$ apodized profile of refractive-index change. Figure 6 shows the reconstructed profiles of the envelope of index change, $\delta n(z)$, and the local Bragg wavelength, $\lambda_{B}(z)$, along the fiber grating. The grating length was cal-
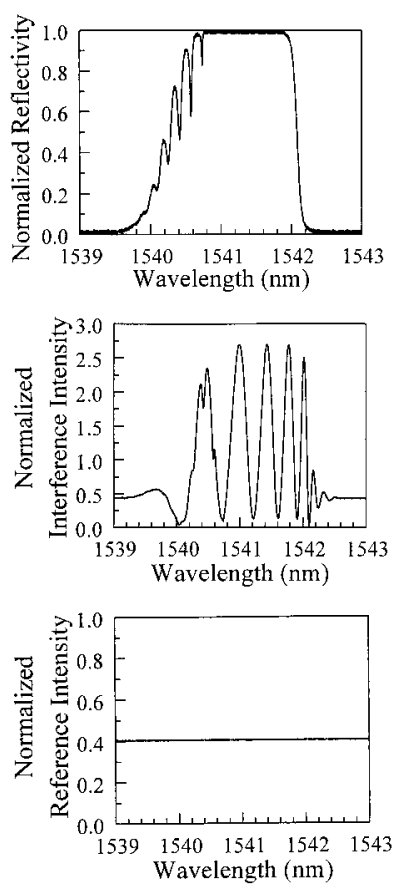

Fig. 3. Measured values of $I_{\text {reflect }}, I_{\text {inter }}$, and $I_{\text {ref }}$ of the chirped grating. 

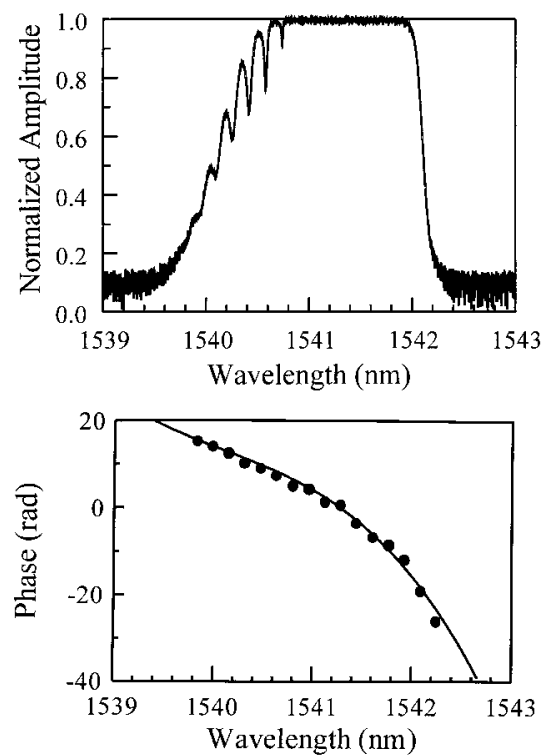

Fig. 4. Retrieved amplitude and phase of the reflection coefficient of the chirped grating.

ibrated to be approximately $6.2 \mathrm{~mm}$. The dc component of the index change, $b \delta n(z)$, was calculated and $b=1.03$ was fitted. The nonconstant dc component of the index change, which caused the self-chirping in the reflectivity spectrum, was verified. The results are in good agreement with the fabrication conditions.

We then consider a fiber grating made with a uniform phase mask. To demonstrate the capability of our reconstruction technique, we purposely fabricated the grating with an UV laser of two peaks in beam profile. Such a laser beam came from an excimer laser, which oscillated with a higher-order transverse mode. Therefore, we expect a depression
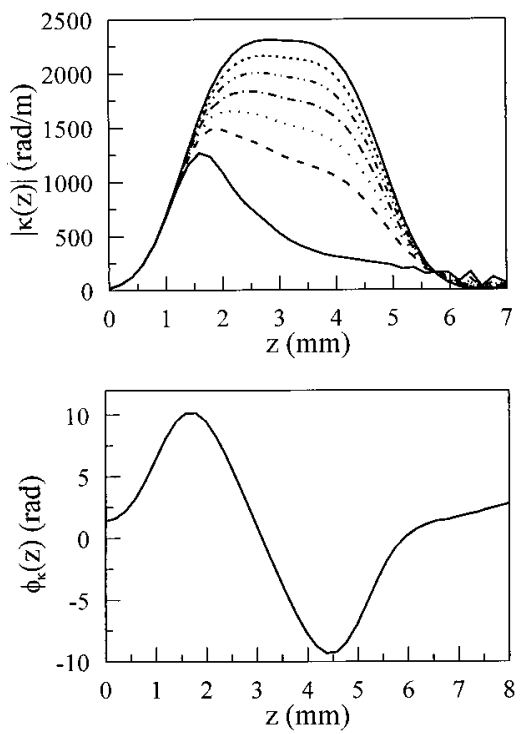

Fig. 5. Amplitude and phase of the reconstructed coupling coefficient of the chirped grating as functions of position in the grating.
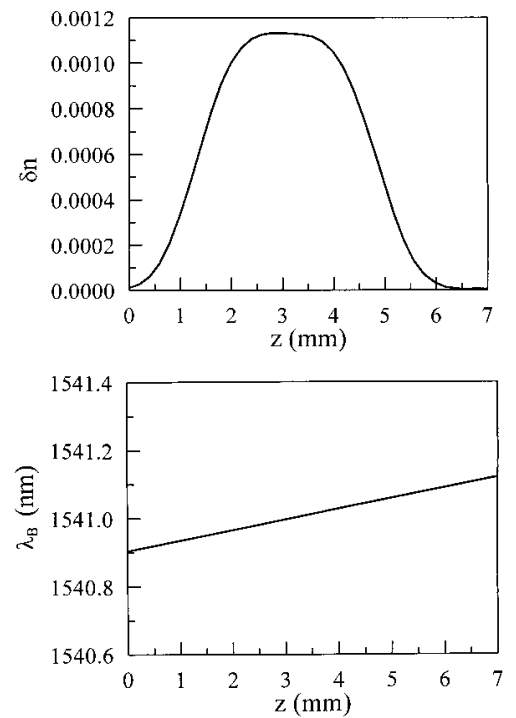

Fig. 6. Reconstructed profiles of the envelope of index change and the local Bragg wavelength along the chirped grating.

of index modulation along the grating. Figure 7 shows the measured values of $I_{\text {reflect }}, I_{\text {inter }}$, and $I_{\text {ref }}$ of this grating. We can see that the measured reflection spectrum is asymmetric, which is contrary to what is expected from a uniform grating but is consistent with the expected index modulation depression. Figure 8 shows the retrieved amplitude and phase of the reflection coefficient. Figure 9 shows the amplitude and phase of the reconstructed coupling coefficient as functions of position in the grating. The result indicates a depression of the UV
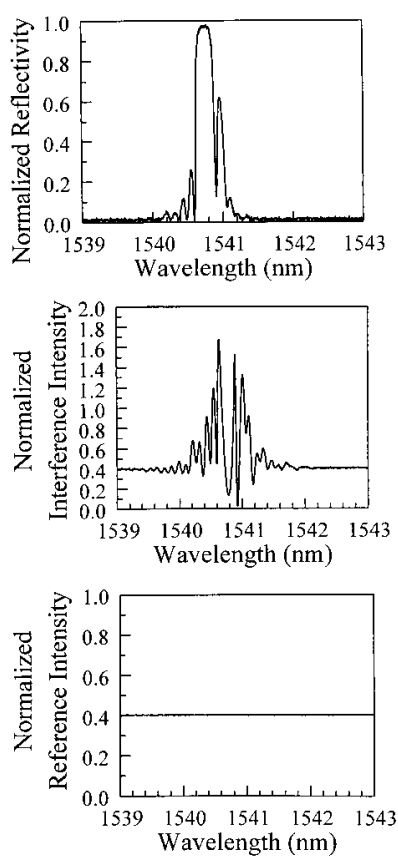

Fig. 7. Measured values of $I_{\text {reflect }}, I_{\text {inter }}$, and $I_{\text {ref }}$ of the uniform grating. 

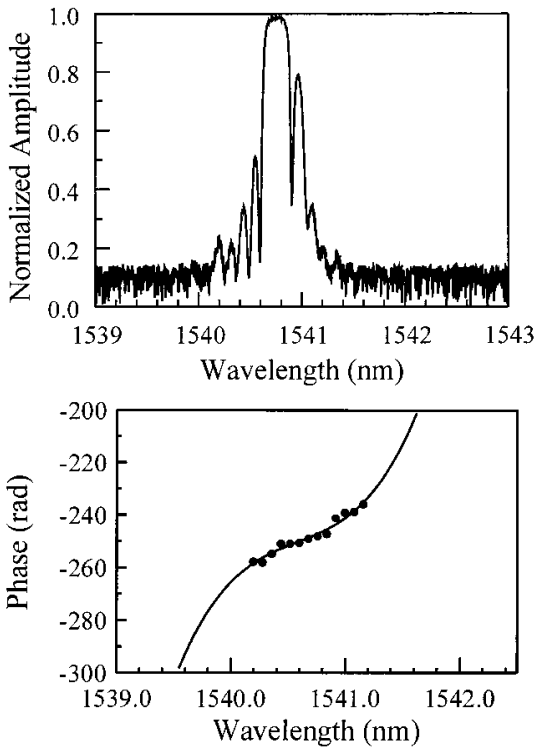

Fig. 8. Retrieved amplitude and phase of the reflection coefficient of the uniform grating.

laser beam profile and of the index modulation, as expected. Figure 10 shows the reconstructed profiles of the envelope of index change and the local Bragg wavelength along the fiber grating. The constant Bragg wavelength near $1540.7 \mathrm{~nm}$ is consistent with the period of the phase mask. We can clearly see the index modulation depression near $z=3 \mathrm{~mm}$, which agrees well with the laser beam profile. The dc component of the index change in this fiber grating was calculated as $b=1$.

\section{Discussions and Conclusion}

The reconstruction algorithm is based on the coupledmode equations and can be simplified by using Fou-
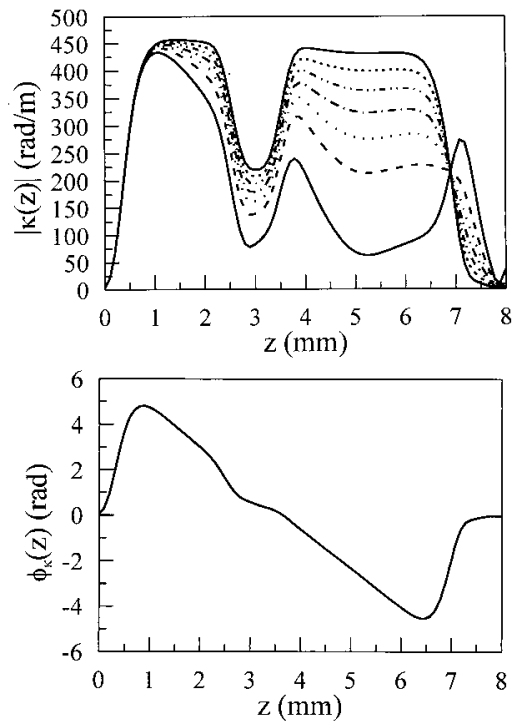

Fig. 9. Amplitude and phase of the reconstructed coupling coefficient of the uniform grating as functions of position in the grating.
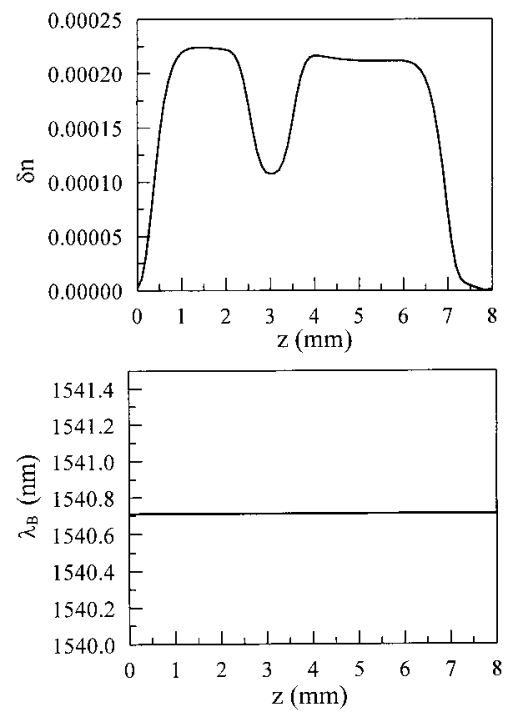

Fig. 10. Reconstructed profiles of the envelope of index change and the local Bragg wavelength along the uniform grating.

rier transformation. Because of the inherent characteristics of the Fourier transformation, the spatial resolution of the reconstructed index profile with this algorithm is limited by the measured spectral bandwidth. The spatial resolution $\Delta z$ can be approximately expressed as

$$
\Delta z=\frac{0.441 \lambda_{B}^{2}}{\Delta \lambda} .
$$

Here, $\Delta \lambda$ is the spectral bandwidth of measurement and $\lambda_{B}$ is the Bragg wavelength. Equation (17) implies that the wider the spectral bandwidth, the higher the resolution of the index profile. For example, when the spectral bandwidth is $4 \mathrm{~nm}$ (as is the case in Fig. 3), the spatial resolution of the reconstructed index profile is approximately $0.26 \mathrm{~mm}$.

The algorithm fails when there is an abrupt change in the index profile or Bragg wavelength of a grating. Another limitation of this method is that the envelope of index change cannot be so strong that signal penetration into the grating is short. Although an additional measurement from the other side of the fiber grating can help to relieve this limitation, ${ }^{8}$ strong Bragg reflection at the edges of a grating still hinders our measurement and reconstruction of the index structure at the middle of the fiber grating.

In summary, we have demonstrated a novel technique for retrieval of the refractive-index profile of a fiber grating with any well-behaved chirp, apodization, and de structures. Two purposely designed fiber gratings were used to demonstrate the capability of this technique. Currently, we are applying this method for the in situ study of the evolution of the index profiles of fiber gratings during the fabrication process.

This research was supported by the National Science Council, the Republic of China, under grants NSC 88-2215-E-002-012, NSC 87-2215-E-002-006, and NSC 87-2215-E-002-007. The help of Gang- 
Chih Lin with the fabrication of the fiber grating is appreciated.

\section{References}

1. L. Zhang, K. Sugden, J. A. R. Williams, I. Bennion, D. C. J. Reid, and C. M. Ragdale, "Postfabrication exposure of gap-type bandpass filters in broadly chirped fiber gratings," Opt. Lett. 20, 1927-1929 (1995).

2. G. Lenz, B. J. Eggleton, C. R. Giles, C. K. Madsen, and R. E. Slusher, "Dispersive properties of optical filters for WDM systems," IEEE J. Quantum Electron. 34, 1390-1402 (1998).

3. G. C. Lin, L. Wang, C. C. Yang, M. C. Shih, and T. J. Chuang, "Thermal performance of metal-clad fiber Bragg grating sensors," IEEE Photon. Technol. Lett. 10, 406-408 (1998).

4. K. Ennser, M. N. Zervas, and R. I. Laming, "Optimization of apodized linearly chirped fiber gratings for optical communications," IEEE J. Quantum Electron. 34, 770-778 (1998).

5. J. A. R. Williams, L. A. Everall, I. Bennion, and N. J. Doran, "Fiber Bragg grating fabrication for dispersion slope compensation," IEEE Photon. Technol. Lett. 8, 1187-1189 (1996).

6. P. A. Krug, R. Stolte, and R. Ulrich, "Measurement of index modulation along an optical fiber Bragg grating," Opt. Lett. 20, 1767-1769 (1995).
7. J. Canning, D. C. Psaila, Z. Brodzeli, A. Higley, and M. Janos, "Characterization of apodized fiber Bragg gratings for rejection filter applications," Appl. Opt. 36, 9378-9382 (1997).

8. E. Brinkmeyer, "Simple algorithm for reconstructing fiber gratings from reflectometric data," Opt. Lett. 20, 810-812 (1995).

9. L. Poladian, "Group-delay reconstruction for fiber Bragg gratings in reflection and transmission," Opt. Lett. 22, 1571-1573 (1997).

10. A. Carballar and M. A. Muriel, "Phase reconstruction from reflectivity in fiber Bragg gratings," J. Lightwave Technol. 15, 1314-1322 (1997).

11. D. Pastor and J. Capmany, "Experimental demonstration of phase reconstruction from reflectivity in uniform fiber Bragg gratings using the Wiener-Lee transform," Electron. Lett. 34, 1344-1345 (1998).

12. T. Edogan, "Fiber grating spectra," J. Lightwave Technol. 15, 1277-1294 (1997).

13. B. C. Collings, K. Bergman, S. T. Cundiff, S. Tsuda, J. N. Kutz, J. E. Cunningham, W. Y. Jan, M. Koch, and W. H. Knox, "Short cavity erbium/ytterbium fiber lasers mode-locked with a saturable Bragg reflector," IEEE J. Sel. Top. Quantum Electron. 3, 1065-1075 (1997). 